\title{
OBTENCIÓN DE UNA CONFIGURACIÓN DE CIRCUITO ELECTRÓNICO BASADO EN SEMICONDUCTORES III-V PARA APLICACIONES ALREDEDOR DE LA FRECUENCIA DE 20 MHz
}

\author{
OBTAINING AN ELECTRONIC CIRCUIT BASED IN III-V SEMICONDUCTOR APPLICATION \\ AROUND THE FREQUENCY OF $20 \mathrm{MHz}$
}

Jhon Jairo Prías Barragán. ${ }^{1,2}$, Ana María Tamayo Ocampo ${ }^{1,3}$, Liliana Tirado Mejía. ${ }^{1}$ y Hernando Ariza-Calderón. ${ }^{1}$

\author{
${ }^{1}$ Laboratorio de Optoelectrónica. \\ ${ }^{2}$ Programa de Tecnología en Electrónica. \\ ${ }^{3}$ Programa de Ingeniería Electrónica, Universidad del Quindío. Armenia, Colombia \\ jjprias@uniquindio.edu.co, anis721@hotmail.com, litirado@uniquindio.edu.co, heariza@uniquindio.edu.co
}

Recibido: Marzo 5 de 2012

Aceptado: Mayo 14 de 2012

*Correspondencia del autor: Laboratorio de Optoelectrónica, Universidad del Quindío. Armenia, Colombia.

jjprias@uniquindio.edu.co

\begin{abstract}
RESUMEN
En este trabajo se presenta la obtención de una configuración de circuito electrónico basado en GaSb, para una aplicación de filtro sintonizado alrededor de $20 \mathrm{MHz}$. Se implementó y automatizó la técnica de espectroscopia de amplitud y fase para el estudio de sistemas metal-semiconductor-metal, en un rango de frecuencia de medida comprendida desde $0.15 \mathrm{MHz}$ hasta $60 \mathrm{MHz}$ y un rango de amplitudes de voltaje comprendido desde $0 \mathrm{mV}$ hasta $500 \mathrm{mV}$. Se hizo el estudio de diagramas de amplitud y fase en muestras de GaSb y GaSb/ $\mathrm{GaSb}$, encontrándose un estrecho pico de resonancia alrededor de $23 \mathrm{MHz}$, el cual fue modelado teóricamente mediante un circuito eléctrico equivalente basado en filtros sintonizados. A partir de este comportamiento eléctrico se diseñó e implementó una aplicación de circuito filtro, con una respuesta sintonizada alrededor de la frecuencia de $20 \mathrm{MHz}$.
\end{abstract}

PALABRAS CLAVES: Amplificador, espectroscopia eléctrica, filtros sintonizados, semiconductores III-V

\begin{abstract}
In this work, obtaining an electronic circuit configuration based on GaSb, for an application filter tuned about $20 \mathrm{MHz}$ was implemented and automated technique of amplitude and phase spectroscopy to study metalsemiconductor-metal systems, at a measuring frequency range comprised from $0.15 \mathrm{MHz}$ to $60 \mathrm{MHz}$ and a voltage amplitude range comprised from $0 \mathrm{mV}$ to $500 \mathrm{mV}$. Was the study of amplitude and phase diagrams in samples of GaSb and GaSb / GaSb, being a narrow resonance peak around $23 \mathrm{MHz}$, which was modeled theoretically by an equivalent electrical circuit tuned filters based. From this electrical behavior was designed and implemented an application of the filter circuit, with a response frequency tuned around $20 \mathrm{MHz}$
\end{abstract}

KEYWORDS: Amplificador, espectroscopia eléctrica, filtros sintonizados, semiconductores III-V 


\section{INTRODUCCIÓN}

La ciencia de los materiales juega un importante papel en el desarrollo tecnológico y en especial en la ingeniería de dispositivos electrónicos, que ha basado su desarrollo en tecnologías tales como la del Si (1) y la del GaAs (2), entre otros importantes nuevos materiales (3). El diseño y fabricación de dispositivos a partir de materiales semiconductores ha sido posible gracias a la amplia labor de investigación científica y tecnológica sobre el tema. Sus usos van desde detectores de rayos $\mathrm{X}$ y circuitos integrados hasta láseres semiconductores con emisión en diferentes longitudes de onda (4). Recientemente ha venido creciendo el interés por el estudio y desarrollo de materiales semiconductores para configuraciones de circuitos electrónicos en aplicaciones de radiofrecuencia (5). Estos trabajos buscan incrementar el ancho de banda, la velocidad de respuesta y la relación señal a ruido en sistemas de comunicación. Por otro lado, se conoce que entre los semiconductores III-V, el GaSb ha sido utilizado en el desarrollo de dispositivos electrónicos tales como láseres, transistores unipolares y bipolares, celdas solares tándem, celdas termo-fotovoltaicas y nanoestructuras cuánticas (6). Sin embargo, hasta donde conocemos, no existen reportes acerca de la utilización del GaSb en configuraciones de circuitos para aplicaciones en radiofrecuencia. En este trabajo presentamos la respuesta en frecuencia de la amplitud y la fase en monocristales de GaSb y homoestructuras de $\mathrm{GaSb}(\mathrm{GaSb} / \mathrm{GaSb})$, para un rango de valores de frecuencia variando desde 0.15 hasta $60 \mathrm{MHz}$ y obteniéndose una resonancia alrededor de los $23 \mathrm{MHz}$.

Para describir teóricamente este comportamiento, se propuso un circuito equivalente, se encontraron las expresiones que describen la dependencia de la amplitud y la fase con la frecuencia y se confrontaron con los resultados experimentales obtenidos. Del respectivo ajuste entre lo medido y lo obtenido teóricamente se encontraron valores para frecuencias de resonancia, factores de calidad y anchos de banda. Además, presentamos una configuración de circuito electrónico de un filtro resonante utilizando las muestras estudiadas.

\section{MATERIALES Y MÉTODOS}

\section{Semiconductores III-V}

Estos materiales han sido ampliamente utilizados en el desarrollo de dispositivos electrónicos y optoelectrónicos, debido a su alta movilidad de electrones, mayor rendimiento en velocidad y menor consumo de energía y tensión (6). El parámetro de red y la energía de banda prohibida pueden escogerse según las características de los materiales y los requerimientos del dispositivo (7). A partir de los metales de los grupos III y V de la tabla periódica se pueden obtener compuestos binarios, GaAs, GaP o GaSb, ternarios, AlGaAs o GaInP, y cuaternarios, GaInNAs o GaInAsSb, entre otros (8).

Estos compuestos se fabrican empleando métodos bien conocidos por la ingeniería de materiales (8). Las muestras estudiadas en este trabajo fueron monocristales comerciales y homoestructuras de GaSb en donde las películas fueron crecidas por epitaxia en fase líquida. En la Tabla I se presentan sus principales características.

\section{Caracterización Eléctrica}

Se utilizó la técnica de caracterización eléctrica mediante diagramas de amplitud y fase (CEDAF) o diagramas de Bode para determinar el comportamiento en frecuencia de las muestras bajo estudio [9, 10]. Esta técnica de caracterización no requiere de preparación previa de las muestras, permite un mejor ancho de banda que los impedancímetros comerciales, permite normalizar los datos de amplitud, es independiente de la función de transferencia del sistema de medida, es de fácil implementación y bajo costo, y ofrece la misma información que la espectroscopia de impedancia eléctrica (11).

En la implementación de la técnica CEDAF se diseñó y construyó un porta-muestra con blindaje tipo jaula de Faraday. En la Figura 1 se presenta el diagrama de bloques de la técnica de CEDAF. Se puede observar que el porta-muestra va conectado al generador de señales de radiofrecuencia y al osciloscopio para la medición de los voltajes de entrada y salida, y de la fase. Estos datos son procesados en un PC de donde se obtienen los diagramas de amplitud y fase.

Las mediciones de los diagramas de amplitud y de fase se realizaron utilizando un generador de radiofrecuencias de la marca Promax GR205, en el que se varía el ancho de banda desde $0.150 \mathrm{MHz}$ hasta $2 \mathrm{GHz}$ y un osciloscopio de la marca Instek de $150 \mathrm{MHz}$, con conectores coaxiales BNC. En la Figura 2 se presenta el circuito equivalente del sistema de medida de la técnica CEDAF. Se puede observar que se trata de un circuito eléctrico en serie alimentado por un generador de voltaje. La impedancia ZM representa el comportamiento eléctrico de la muestra; la resistencia en serie RS utilizada es de $10 \mathrm{~K} \Omega$. 
Tabla 1. Muestras estudiadas

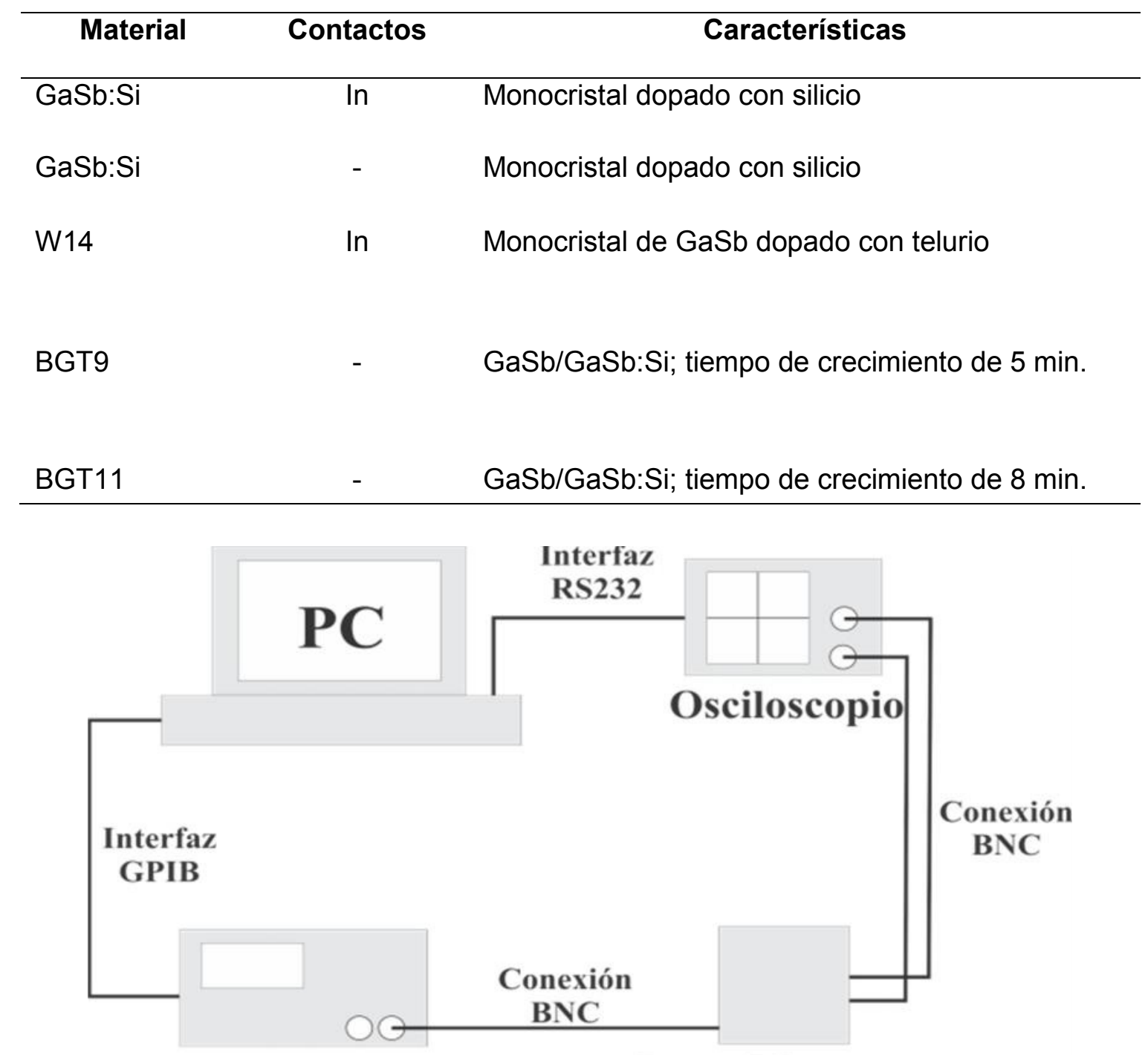

Generador de RF

Figura 1. Diagrama del montaje experimental de la técnica CEDAF.

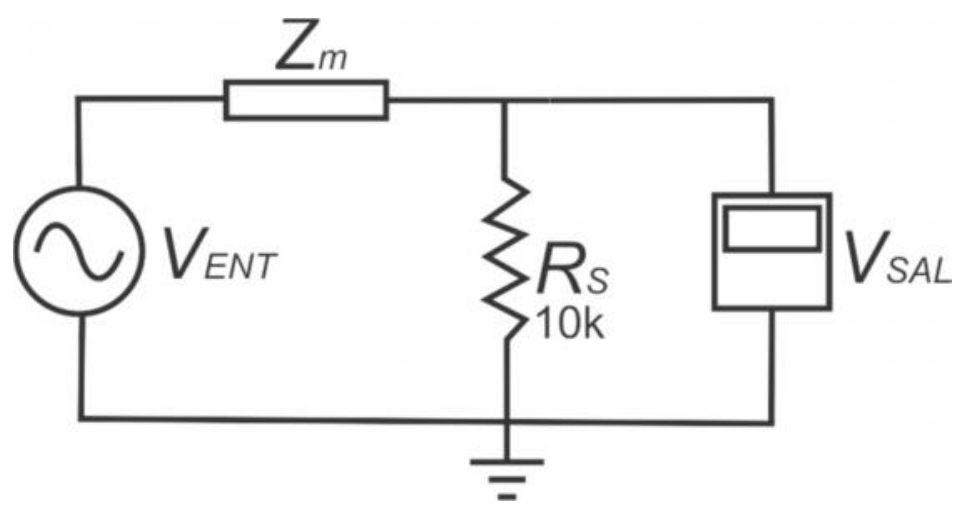

Figura 2. Circuito equivalente de la técnica de CEDAF. 


\section{Teoría de la Técnica de CEDAF}

Las aplicaciones de la técnica de CEDAF son amplias y abarcan tópicos que van desde la ingeniería del control hasta la ingeniería de las comunicaciones. En esta técnica se mide de manera simultánea la amplitud de los voltajes de salida VSAL y de entrada VENT, y la fase $\phi$ para variaciones de la frecuencia $f$ de la señal de entrada, tal como se presenta en el circuito de la Figura 3. La relación entre ambas mediciones nos da la atenuación AV, descrita por:

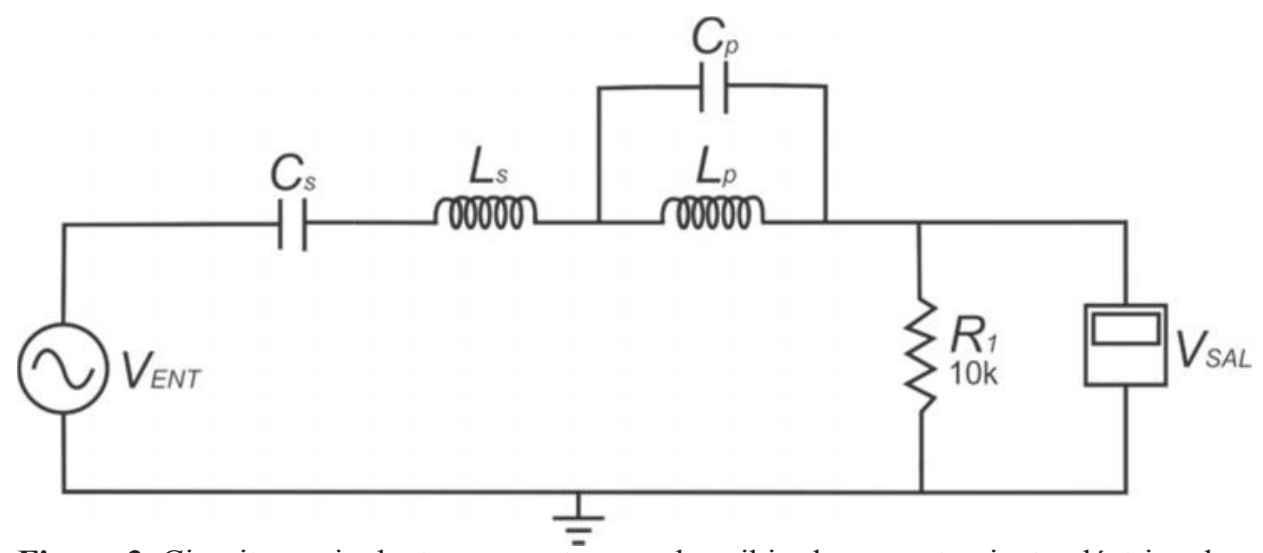

Figura 3. Circuito equivalente propuesto para describir el comportamiento eléctrico de los semiconductores estudiados..

$$
A_{V}(\omega)=\frac{V_{S A L}}{V_{E N T}}
$$

Siendo $\omega=2 \pi f$. Y en términos de las componentes del circuito de la Figura 3, re-escribimos la ecuación (1) como:

$$
A_{V}(\omega)=\frac{R_{S}}{R_{S}+Z_{M}(\omega)}
$$

siendo $Z_{M}(\omega)$ la impedancia dependiente de la frecuencia angular, asociada al comportamiento eléctrico de la muestra.

Al realizar el análisis de la ecuación (2) para diferentes configuraciones de circuitos eléctricos pasivos, se obtienen los comportamientos en frecuencia, tanto para la amplitud $A V(\omega)$ como para la fase $\Phi(\omega)$. De este análisis se obtienen frecuencias de corte y resonancia, factores de calidad y ancho de banda para cada configuración. Los valores obtenidos para el circuito equivalente propuesto, se presentan más adelante.

El análisis de los diagramas de amplitud y fase medidos a las muestras bajo estudio, se realizó considerando dos contribuciones en el comportamiento eléctrico: la primera contribución fue asociada a un circuito filtro pasabanda sintonizado, para valores de frecuencia alrededor de $23 \mathrm{MHz}$, y la segunda contribución fue asociada a un circuito atrapa-banda, para valores de frecuencia alrededor de $35 \mathrm{MHz}$.

En la Figura 3 se presenta el circuito equivalente de las muestras estudiadas, en donde se describe el comportamiento eléctrico con dos contribuciones resonantes, las cuales fueron establecidas por las redes $\mathrm{L}_{\mathrm{S}} \mathrm{C}_{\mathrm{S}}$ en serie y $\mathrm{L}_{\mathrm{P}} \mathrm{C}_{\mathrm{P}}$ en paralelo: la primera se atribuye a efectos del campo eléctrico oscilante en la superficie del material semiconductor, y la segunda, al efecto del campo eléctrico oscilante en el volumen del material semiconductor.

Utilizando el análisis de la ecuación (2) en el circuito de la Figura 3, para el caso de la primera contribución (filtro pasa-banda, $\mathrm{PB}$ ), se puede obtener $A V(\omega)$.

Es decir, la dependencia de la amplitud con la frecuencia, está dada por:

$$
\left|A_{V P B}(\omega)\right|=\sqrt{\frac{\left(\frac{f}{f_{c 1}}\right)^{2}}{\left(\frac{f}{f_{c 1}}\right)^{2}+\left(\left(\frac{f}{f_{o 1}}\right)^{2}-1\right)^{2}}}
$$


donde $f_{C I}=1 /\left(2 \pi R_{S} C_{S}\right)$ es la frecuencia de corte y $f_{01}=1 /$ $\left(2 \pi\left(L_{S} C_{S}\right)^{1 / 2}\right)$ es la frecuencia de resonancia. La dependencia de la fase con la frecuencia, está dada por:

$$
\phi_{P B}=\tan ^{-1}\left[\left(\left(\frac{f}{f_{o 1}}\right)^{2}-1\right) /\left(\frac{f}{f_{c 1}}\right)\right]
$$

De la misma manera, para la segunda contribución (filtro atrapa-banda, $\mathrm{AB}$ ), la dependencia de la amplitud con la frecuencia está dada por:

$$
\left|A_{V A B}(\omega)\right|=\sqrt{\left.\frac{1+\left(\frac{f}{f_{c 2}}\right)^{2}}{\left(\frac{f}{f_{o 2}}\right)^{2}-1}\right)^{2}}
$$

siendo $f_{C 2}=1 /\left(2 \pi R_{P} C_{P}\right)$ la frecuencia de corte y $f_{02}=1 /$ $\left(2 \pi\left(L_{P} C_{P}\right)^{1 / 2}\right)$ la frecuencia de resonancia. La dependencia de la fase con la frecuencia está dada por:

$$
\phi_{A B}(\omega)=\tan ^{-1}\left[\left(\frac{f}{f_{c 2}}\right) /\left(\left(\frac{f}{f_{o 2}}\right)^{2}-1\right)\right]
$$

Por lo tanto, considerando el efecto combinado de las dos contribuciones a la respuesta eléctrica, se obtiene que las dependencias de la amplitud y la fase con la frecuencia de la señal del voltaje de entrada, están dadas por:

$$
\begin{gathered}
\left|A_{V T}\right|=\left|A_{V P B}\right| *\left|A_{V A B}\right| \\
\phi_{T}=\phi_{P B}+\phi_{A B}
\end{gathered}
$$

Las ecuaciones (7) y (8) permitieron la descripción teórica de los diagramas de amplitud y fase tomados experimentalmente en los semiconductores estudiados.

\section{RESULTADOS Y DISCUSIÓN}

Empleando el montaje experimental de la Figura 1, se obtuvieron los diagramas de amplitud y fase presentados en las Figuras 4 y 5. En los diagramas de amplitud se puede observar un agudo pico resonante alrededor del valor de la frecuencia de $23 \mathrm{MHz}$ y en los diagramas de fase se puede observar el máximo desfase de los voltajes, para valores de frecuencia comprendidos entre 23 MHz y $37 \mathrm{MHz}$. En la descripción teórica de los diagramas de amplitud y fase, se utilizaron las ecuaciones (7) y (8) respectivamente, encontrándose una alta concordancia entre la teoría y los resultados experimentales. Para el caso de la dependencia de la fase con la frecuencia, la teoría predice en las bandas de atenuación un desfase menor que el que apareció experimentalmente (Figuras $4 \mathrm{~b}$ y $5 \mathrm{~b}$ ), atribuido a los bajos valores de la amplitud del voltaje de salida para esas regiones.

En la Tabla II se presenta el consolidado de los valores obtenidos del ajuste de los datos experimentales, al aplicar las ecuaciones (7) y (8) a los diagramas de amplitud y fase de las Figuras 4 y 5 . Se puede observar que las muestras, a excepción de la BGT11, presentan un factor de calidad Q mayor que 10, la cual es una característica típica de un circuito resonante de alta selectividad; es decir, un menor ancho de banda. Al comparar los resultados presentados en la Tabla II se puede observar que la presencia de los contactos eléctricos en los monocristales de GaSb:Si incrementa el factor de calidad Q y disminuye el ancho de banda. El valor de capacitancia CS que resulta en el circuito equivalente después de realizar el ajuste a los datos experimentales, es diferente para cada muestra estudiada. Esto se puede atribuir a diferencias en la densidad de defectos superficiales de cada muestra.

El incremento del valor de $L_{S}$ en los monocristales se asocia a la presencia del contacto eléctrico, mientras que la presencia de la película en las homoestructuras contribuye a disminuir de manera significativa este valor. Es decir, la película de GaSb tiene un importante efecto de reducción del valor de $L_{S}$, mientras que para $L_{P}$ se obtiene un mismo valor para el mismo tipo de sustrato, lo que implica una fuerte influencia del tipo de dopaje. De la misma manera, el valor de la capacitancia $C_{P}$, varía con el dopaje.

El efecto resonante observado en estos semiconductores alrededor de los $23 \mathrm{MHz}$ se aprovechó en el diseño e implementación de una configuración de circuito filtro sintonizado, Figura 6, en el cual se puede observar que se trata de una combinación serie - paralelo entre la muestra, la resistencia $R_{S}$, el amplificador y la carga de salida $R_{C}$. 
Tabla 2. Consolidado de parámetros según ajuste teórico.

\begin{tabular}{lrrrrr}
\hline $\begin{array}{c}\text { Parámetros } \\
\text { obtenidos }\end{array}$ & GaSb:Si & \multicolumn{1}{c}{ GaSb:Si* } & \multicolumn{1}{c}{ W14* $^{*}$} & \multicolumn{1}{c}{ BGT9 } & BGT11 \\
\hline$f_{0}(\mathrm{MHz})$ & 23.713 & 22.846 & 22.766 & 22.644 & 22.839 \\
$Q$ & 12.74 & 14.32 & 21.95 & 15.77 & 9.62 \\
$B_{W}(\mathrm{MHz})$ & 1.861 & 1.595 & 1.036 & 1.435 & 2.372 \\
$C_{S}(\mathrm{pf})$ & 0.043 & 0.034 & 0.027 & 0.26 & 0.607 \\
$L_{S}(\mathrm{mH})$ & 1.05 & 1.44 & 1.81 & 0.19 & 0.08 \\
$C_{P}(\mathrm{pf})$ & 0.673 & 0.674 & 0.226 & 0.673 & 0.657 \\
$L_{P}(\mu \mathrm{H})$ & 30 & 30 & 90 & 30 & 30 \\
$R(\mathrm{k} \Omega) \pm 1$ & 10 & 10 & 10 & 10 & 10 \\
$\xi^{2}$ & 0.82 & 0.34 & 0.23 & 0.31 & 1.01 \\
$*$ contactos de In & & & & &
\end{tabular}

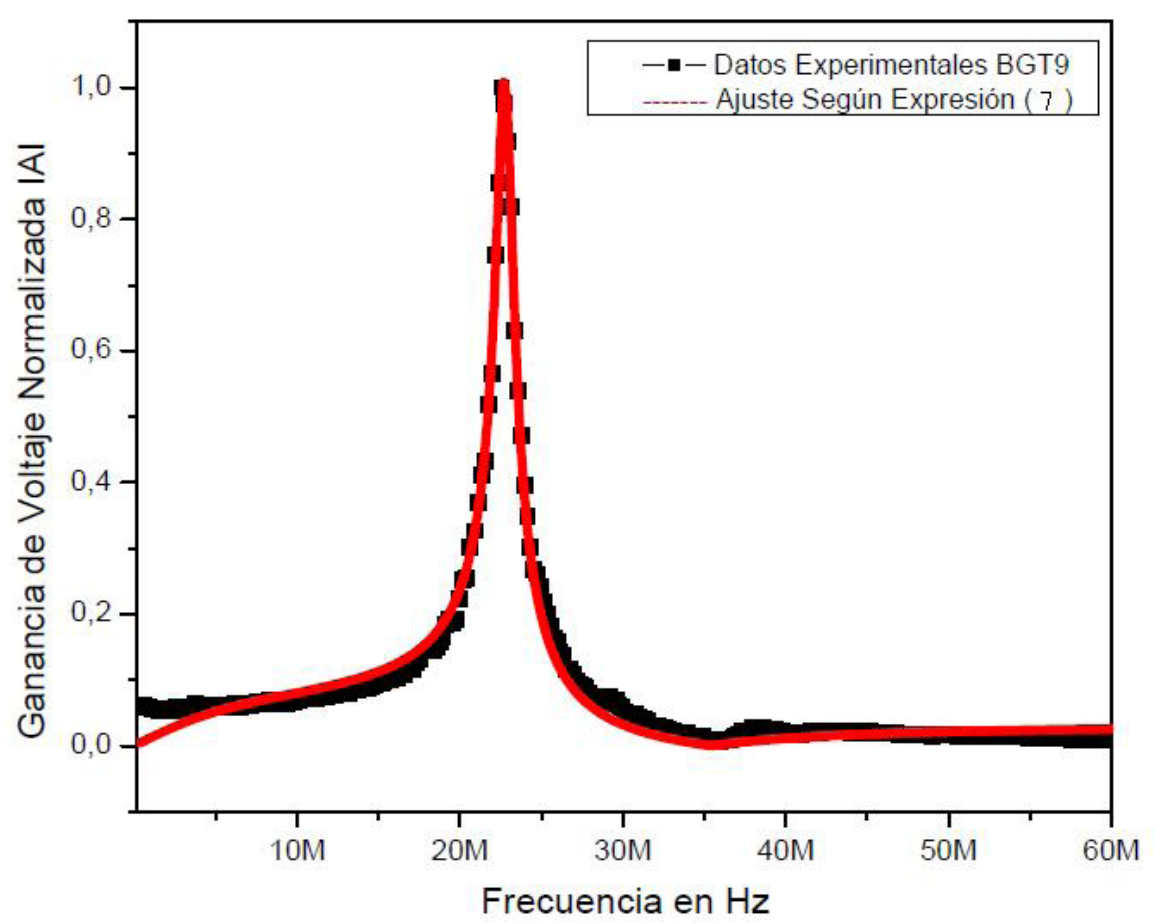

a)

Figura 4. a) Diagrama de amplitud en la homoestructura $\mathrm{GaSb} / \mathrm{GaSb}$ (BGT9). 


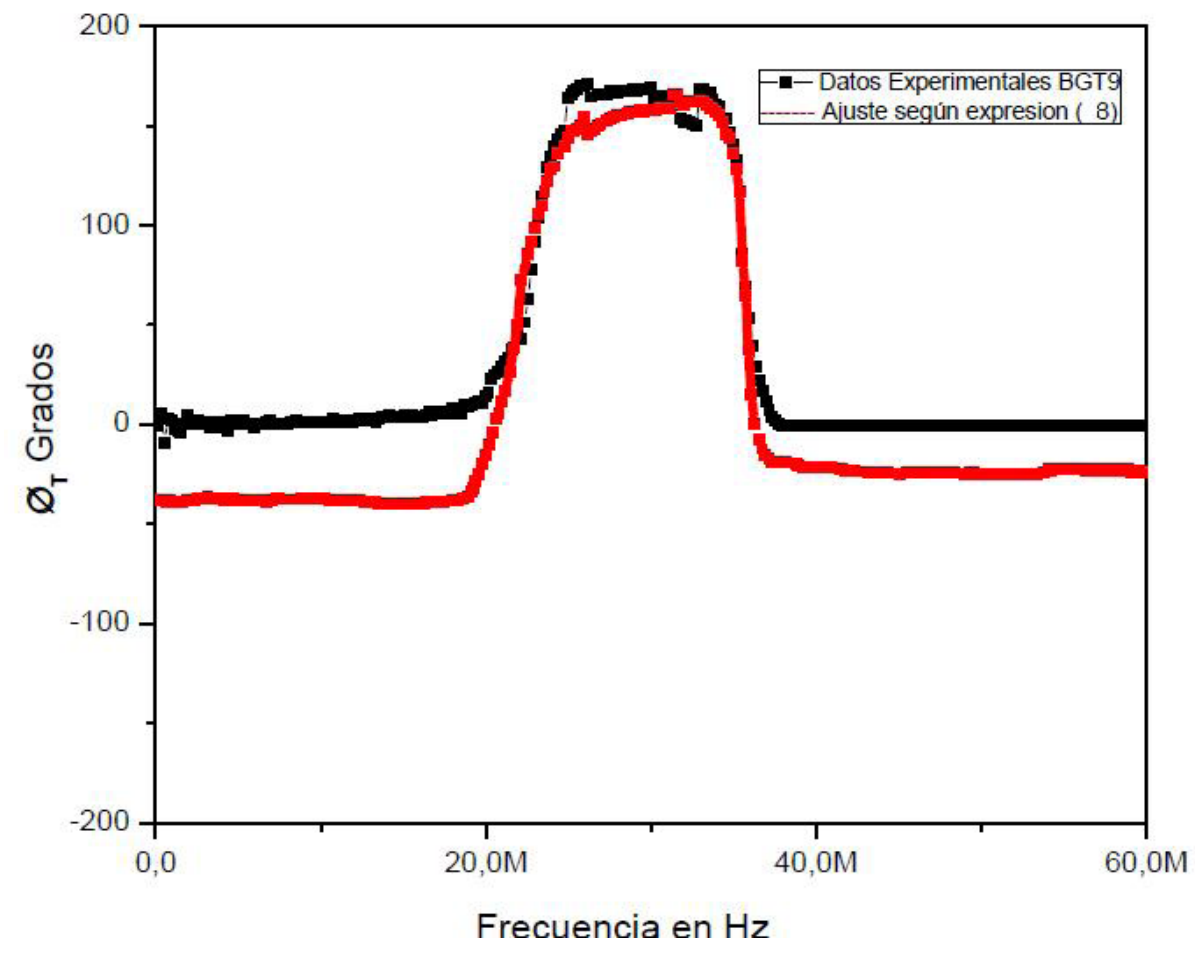

b)

Figura 4. b) Diagrama de fase en la homoestructura GaSb/GaSb (BGT9).

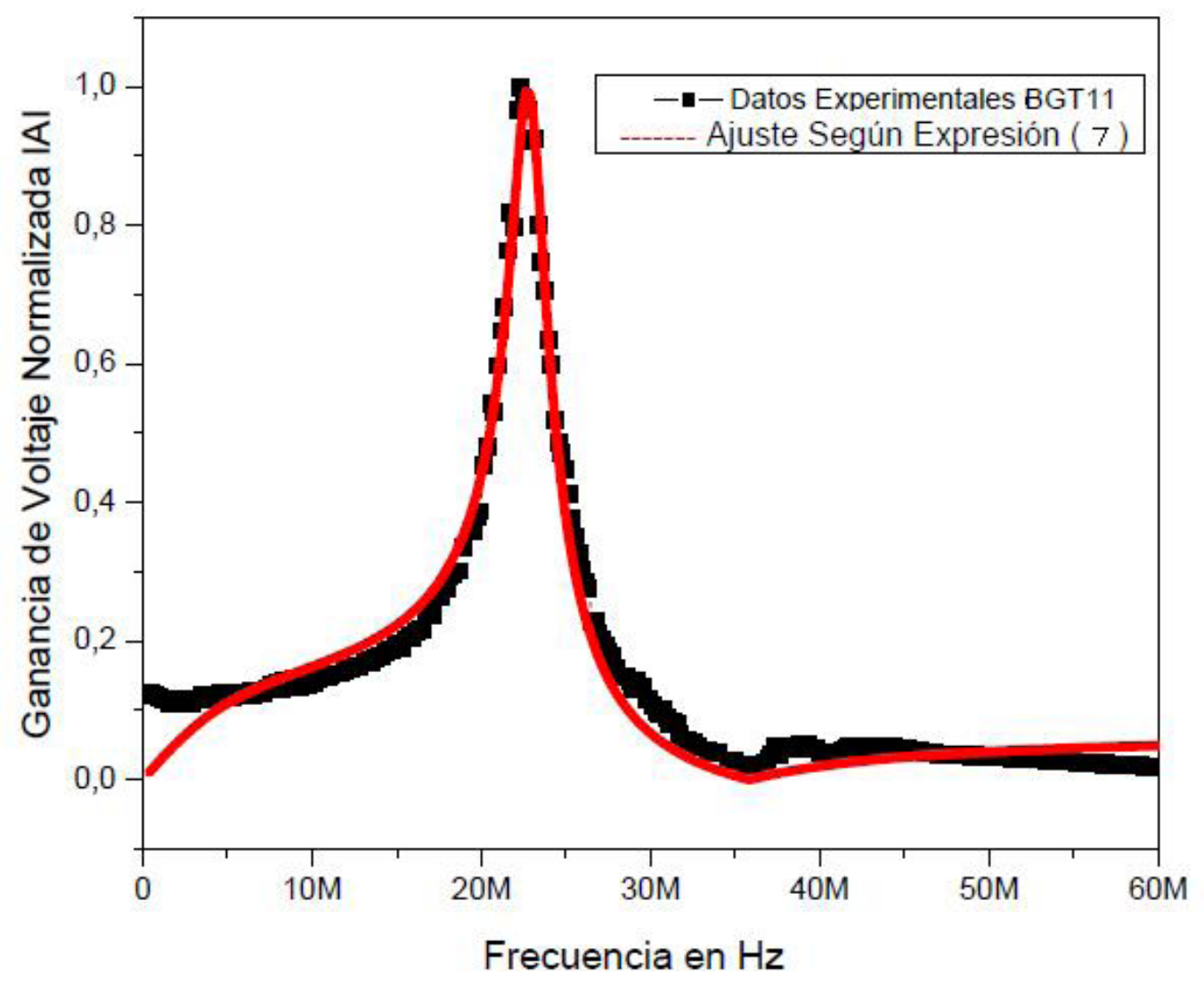

a)

Figura 5. a) Diagrama de amplitud en la homoestructura GaSb/GaSb (BGT11). 


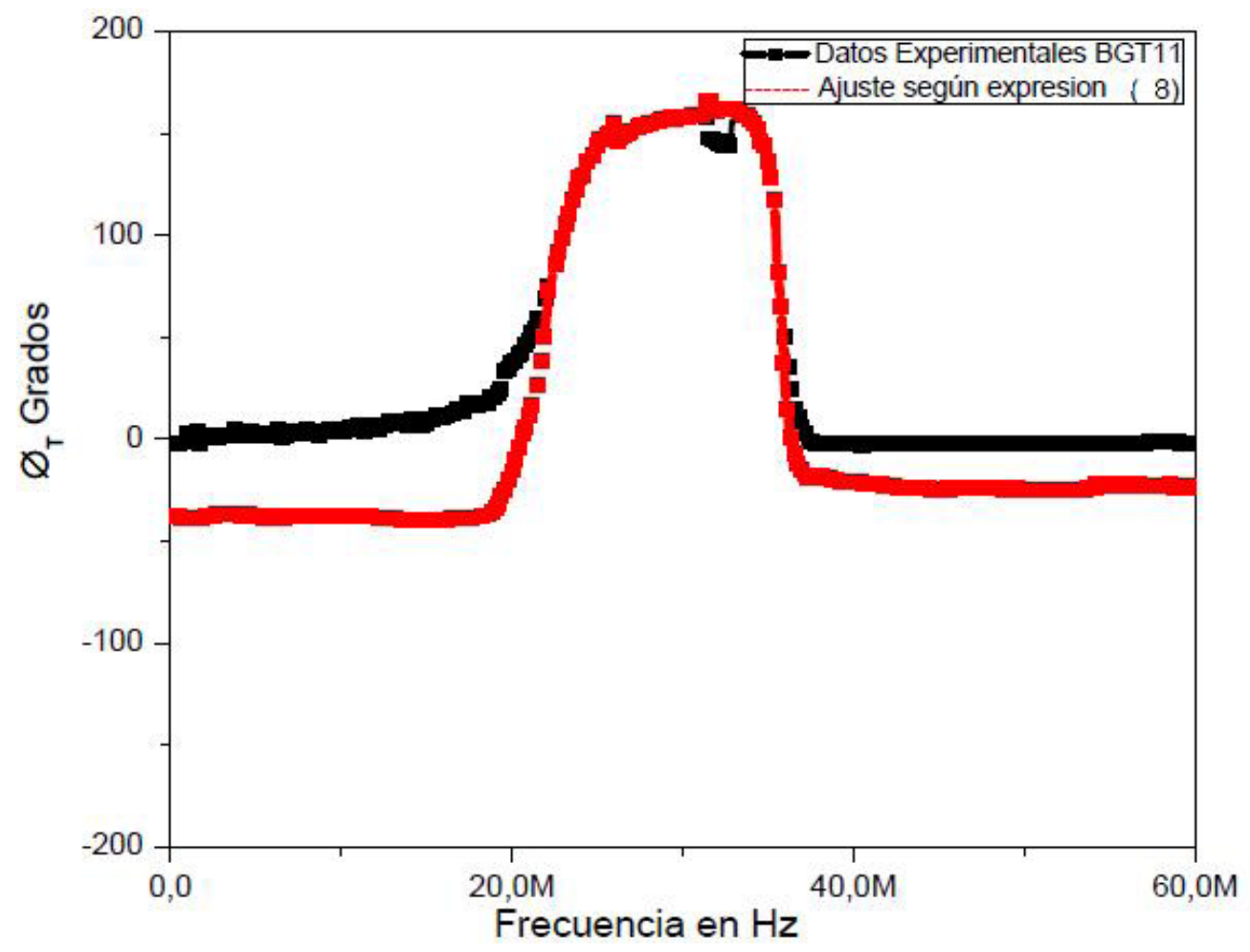

b)

Figura 5. b) Diagrama del comportamiento en frecuencia de la fase en la homoestructura $\mathrm{GaSb}$ / GaSb (BGT11).

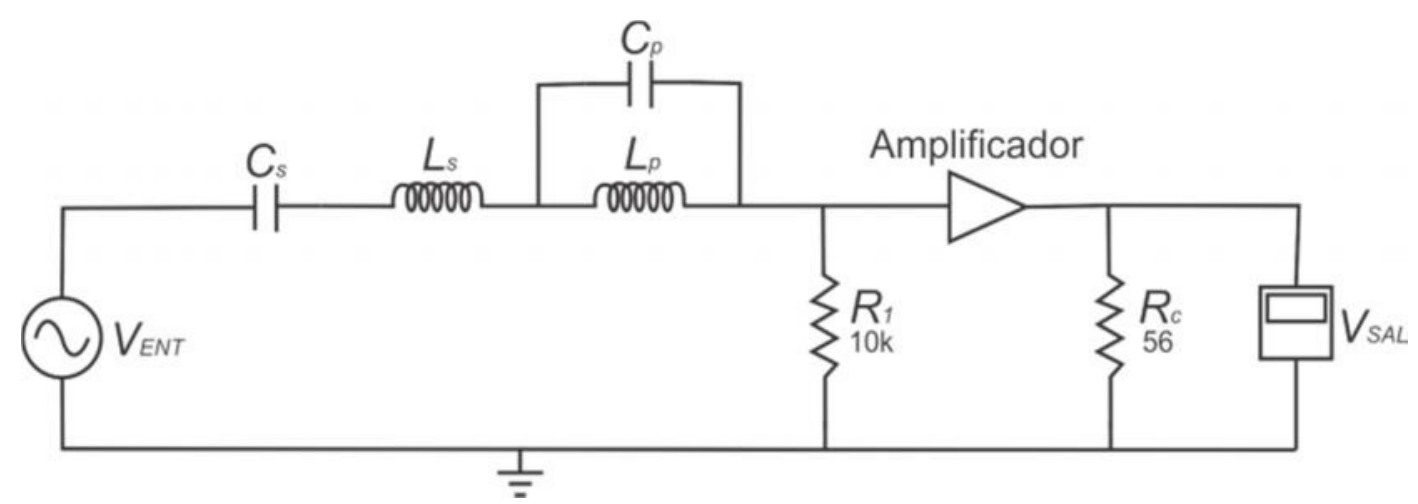

Figura 6. Circuito equivalente de la aplicación propuesta en este trabajo. 
En la Figura 7a) se presenta la respuesta en frecuencia, tanto para la amplitud como para la fase del circuito de la Figura 6. Se puede observar que la presencia del material semiconductor disminuye el valor de la frecuencia de resonancia y el ancho de banda, debido al acople de impedancias.

En la Figura 7 b) se puede observar que cuando se tiene el pico resonante, la fase se acerca al valor de cero grados, en ambas configuraciones, tal como era de esperarse. Proponemos que los comportamientos resonantes encontrados en los semiconductores estudiados, se pueden aprovechar en circuitos electrónicos, tales como osciladores, filtros, codificadores, decodificadores, circuitos triplicadores de frecuencia, antenas, circuitos emuladores y acopladores de impedancia, entre otros $(12,13)$. Además, proponemos el circuito de la Figura 7 para ser utilizado como herramienta de diagnóstico de redes de internet, entre otras aplicaciones.

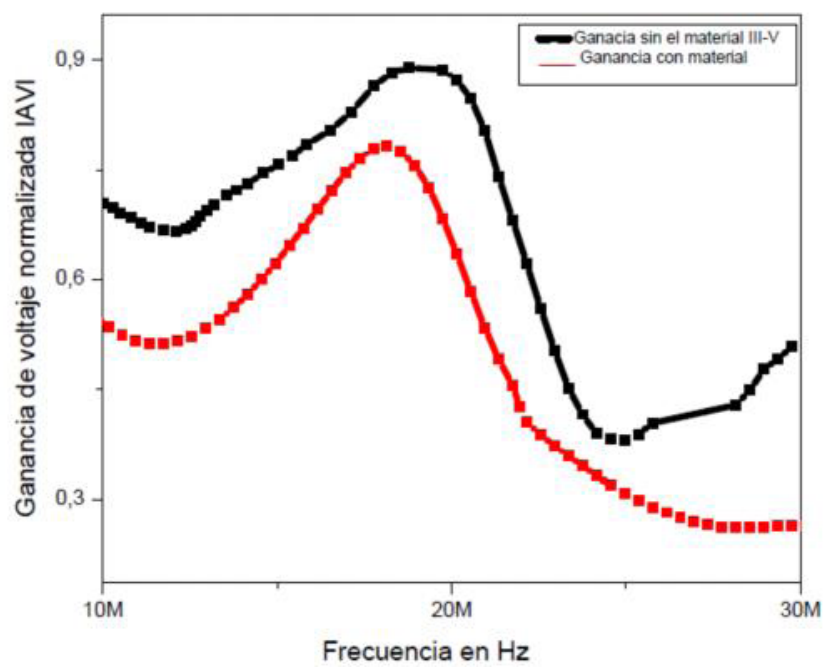

a)

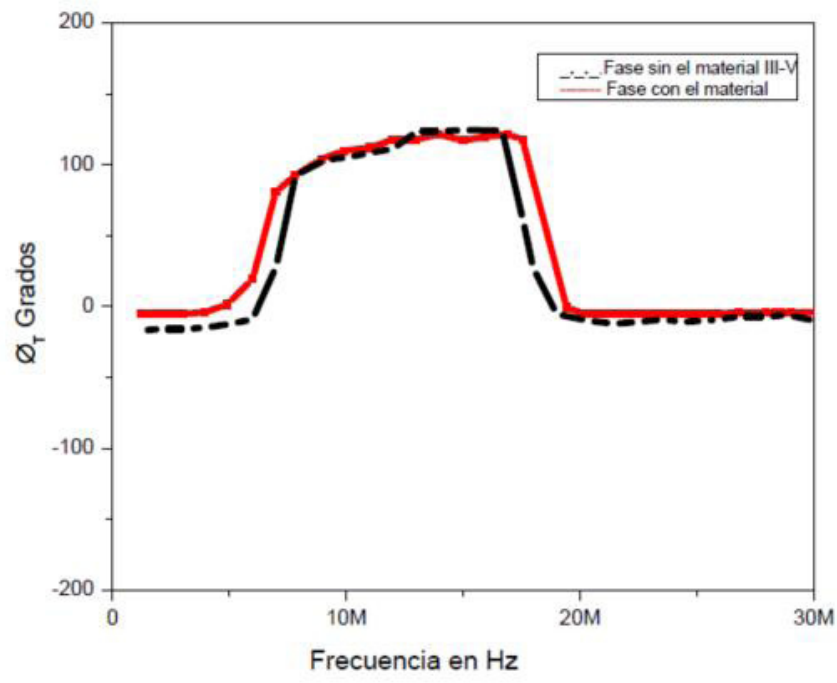

b)

Figura 7. Comportamiento en frecuencia de la configuración de circuito propuesto en este trabajo, a) Diagrama de amplitud y b) Diagrama de fase. 


\section{CONCLUSIONES}

Hemos obtenido una configuración de circuito electrónico basado en semiconductores III-V, particularmente GaSb, para aplicaciones alrededor de la frecuencia de $20 \mathrm{MHz}$. Se implementó, automatizó y calibró la técnica de CEDAF, para el estudio de la respuesta en frecuencia de sistemas metal-semiconductor-metal, empleando GaSb. Se encontró que los monocristales de GaSb y las homoestructuras de $\mathrm{GaSb} / \mathrm{GaSb}$, exhiben efectos resonantes alrededor de $23 \mathrm{MHz}$ y $35 \mathrm{MHz}$, para los efectos de pasa-banda y atrapa-banda, respectivamente. Se encontró un modelo de circuito equivalente basado en la respuesta combinada de circuitos resonantes en serie y en paralelo, para describir los diagramas de amplitud y fase de los sistemas metal-semiconductor-metal, estudiados en este trabajo. La resonancia en serie se asoció a efectos del campo eléctrico oscilante en la superficie, mientras que la resonancia en paralelo, se atribuyó a efectos del campo eléctrico oscilante en el volumen de las muestras estudiadas. Finalmente, proponemos la metodología empleada en este trabajo para extender las aplicaciones de los semiconductores $\mathrm{GaSb}$ y $\mathrm{GaSb} / \mathrm{GaSb}$ del campo de la optoelectrónica al campo de la electrónica especialmente alrededor de los $23 \mathrm{MHz}$.

\section{AGRADECIMIENTOS}

Este trabajo ha sido apoyado por la Universidad del Quindío.

\section{BIBLIOGRAFÍA}

1. F. Sandoval Ibarra. El silicio y sus propiedades como material sensor. Revista Mexicana de Física, 2001; 47(2), pp. 107-115.

2. Onur Esame, Yasar Gurbuz, Ibrahim Tekin, Ayhan Bozkurt. Performance comparison of state-ofthe-art heterojunction bipolar devices(HBT) based on AlGaAs/GaAs, Si/SiGe and InGaAs/InP. Microelectronics Journal, 2004; 35, pp. 901-908.

3. J. J. Prías-Barragán, L. Tirado-Mejía, H. Ariza-Calderón, L. Baños, J. J. Perez-Bueno, M. E. Rodríguez. Band gap energy determination by photoacoustic absorption and optical analysis of Cd1xZnxTe for low zincconcentrations. Journal of Crystal Growth, 2006; 286, pp. 279-283.

4. Rallabandi Sailaja, Prem B. Bisht. Tunable multiline distributed feedback dye laser based on the phenomenon of excitation energy transfer. Organic Electronics, 2007; 8, pp. 175-183.

5. K. F. Renk, B. I. Stahl. Operation of a semiconductor superlattice oscillator. Physics Letter A, 2011; 375, pp. 2644-2641.

6. J. Tousková, et al. Current-voltage characteristics of GaSb homojunctions prepared by MOVPE". Solid-State Electronics, 2011; 47, pp. 1471-1478.

7. H. Hasegawa, M. Akazawa, A. Domanowska, B. Adamowicz. Surface passivation of III-V semiconductors for future CMOS devices-Past research, present status and key issues for future. Applied Surface Science, 2010; 256, pp. 5698-5707.

8. Chao Jiang, Takuya Kawazu, Shigeki Kobayashi, Hiroyuki Sakaki. Molecular beam epitaxial growth of very large lateral anisotropic $\mathrm{GaSb} / \mathrm{GaAs}$ quantum dots. Journal of Crystal Growth, 2007; 301-302,pp. 828-832.

9. Mahmood Ghanbari, Mohammad Haeri. Order and pole locator estimation in fractional order systems using bode diagram". Signal Processing, 2010; 91, pp. 191-202.

10. M. Kheirmand, A. Asnafi. Analytic parameter identification of proton exchange membrane fuel cell catalyst layer using electrochemical impedance spectroscopy". International Journal of Hydrogen Energy, 2011; 36, pp. 13266-13271.

11. Chi-Min Lin, Lung-Hsiang Chen, Tse-Min Chen. The development and application of an electrical impedance spectroscopy measurement system for plant tissues. Computers and Electronics in Agriculture, 2012; 82, pp. 96-99.

12. E. Zampetti, S. Pantalei, A. Macagnano, E. Proietti, C. Di Natale, A. D'Amicob. Use of a multiplexed oscillator in a miniaturized electronic nose basedon a multichannel quartz crystal microbalance. Sensors and Actuators B, 2008; 131, pp. 159-166.

13. Ali ÜmitKeskina, Kirat Pal, ErhanHancioglu. Resistorless first-order all-pass filter with electronic tuning. Int. J. Electron. Commun. (AEÜ), 2008; 62, pp. $304-306$. 